\title{
LEARNING FROM IMAGES AND SPEECH WITH NON-NEGATIVE MATRIX FACTORIZATION ENHANCED BY INPUT SPACE SCALING
}

\author{
Joris Driesen $^{1}$, Hugo Van hamme ${ }^{1}$, W. Bastiaan Kleijn ${ }^{2}$ \\ ${ }^{1}$ Dept. ESAT-PSI, K.U.Leuven, Leuven, Belgium \\ ${ }^{2}$ Sound and Image Processing Laboratory, KTH, Stockholm, Sweden \\ joris.driesen@esat.kuleuven.be, hugo.vanhammedesat.kuleuven.be, bastiaan@kth.se
}

\begin{abstract}
Computional learning from multimodal data is often done with matrix factorization techniques such as NMF (Nonnegative Matrix Factorization), pLSA (Probabilistic Latent Semantic Analysis) or LDA (Latent Dirichlet Allocation). The different modalities of the input are to this end converted into features that are easily placed in a vectorized format. An inherent weakness of such a data representation is that only a subset of these data features actually aids the learning. In this paper, we first describe a simple NMF-based recognition framework operating on speech and image data. We then propose and demonstrate a novel algorithm that scales the inputs of this framework in order to optimize its recognition performance.
\end{abstract}

Index Terms: Machine Learning, Vocabulary Acquisition, Image Recognition, Multi-modal Learning, Feature Selection.

\section{INTRODUCTION}

Language learning is a process that encompasses more than just recognizing recurring patterns in speech. In order for discovered word-like patterns to have any meaning or usability, they have to be associated with certain objects or concepts in other modalities than speech. By concepts we mean anything that can be clearly perceived, yet is not an object (e.g. soft, sour, smelly, etc). A learning agent that is unable to see, smell, taste or touch will be unable to acquire language skills. For this reason, there is a growing interest in systems that perform learning on information from several modalities combined, most notably speech and image $[1,2,3]$. In such systems, aside from linking learned speech patterns with real world objects, the presence of visual data is supposed to facilitate the acquisition of words and vice versa. An often used recognition paradigm that is applied on such data is the factorization of input vectors in non-negative basis vectors [2,3].

The usage of any algorithm that performs this particular task (non-negative matrix factorization [4], probabilistic latent semantic analysis [5], latent Dirichlet allocation [6]) necessitates a vectorized representation of the input data. A well-known example of such a representation is the bag-ofwords model of a text document, which is widely used in applications such as topic detection, document classification, spam filtering, etc. However, for any vector input, the possibility exists that some of its features have little relevance for the application. For example, when using a bag-of-words model it is common practice to disregard articles and other ubiquitous words since they carry little information. However, this is a heuristic operation that requires considerable prior knowledge about the data involved and is all but impossible to apply on vectorized speech- and image data (see section 4.3). In this paper, we consider NMF to perform recognition on such data, similar to work presented in [3]. The novelty of this paper is the algorithm introduced within this recognition framework, that automatically determines not only the importance of both modalities for the task at hand, i.e. recognizing objects, but also the relative importance of each separate input feature within these two modalities. To achieve this, our approach determines an appropriate scaling for each element of the vectorized representation.

This input scaling approach differs from Automatic Relevance Determination for NMF, which attempts to find the optimal inner dimension of the factorization $([7,8])$. Whereas these methods treat the input data as a given and attempt to find the optimal underlying model order, our algorithm attempts to adjust the input data such that it optimally fits a given model order. At the time of writing, the authors are unaware of other published work in which the same problem is addressed.

In section 2 of this paper, we describe a general predictive framework for NMF, capable of learning patterns from multimodal data in a supervised way and detecting these patterns in unseen data. In section 3 , we explain how the input weights can be derived in such a way that the accuracy of this predictive framework is optimized. In section 4 we apply this recognition algorithm on images, speech and both modalities combined. All results are obtained both with and without scaling of the input features, confirming that the scaled input features yield models of superior accuracy.

\section{PREDICTION WITH NMF}

NMF is an algorithm that factorizes a non-negative matrix $V$, with input vectors along the columns, of size $M \times N$ into two 
non-negative matrices $W$ and $H$ of respective sizes $M \times R$ and $R \times N(R \ll M$ and $R \ll N)$. This is done by minimizing a distance measure between $V$ and the product $(W H)$. In this paper, we opt for the extended Kullbeck-Leibler divergence [4]:

$$
D_{K L}(V \| W H)=\sum_{i j} V_{i j} \log \frac{V_{i j}}{(W H)_{i j}}+(W H)_{i j}-V_{i j}
$$

which can be minimized iteratively with the multiplicative updates:

$$
\begin{aligned}
W_{i r}^{(k+1)} & =W_{i r}^{(k)} \frac{\sum_{j} \frac{V_{i j} H_{r j}^{(k)}}{\left(W^{(k)} H^{(k)}\right)_{i j}}}{\sum_{j} H_{r j}^{(k)}} \\
H_{r j}^{(k+1)} & =H_{r j}^{(k)} \frac{\sum_{i} \frac{V_{i j} W_{i r}^{(k+1)}}{\left(W^{(k+1)} H^{(k)}\right)_{i j}}}{\sum_{i} W_{i r}^{(k+1)}}
\end{aligned}
$$

in which $k$ is an iteration index. When an optimum is found, the columns of $W$ contain $R$ salient non-negative basis vectors with which the columns of $V$ can be approximately reconstructed using weighted additions.

While NMF is mostly used for structure discovery in nonnegative data, it can also be used for prediction. Suppose we have two data matrices $V^{t r n}$ and $V^{t s t}$, respectively containing training data and testing data. The rows of both these matrices can be separated into three groups: $V_{0}, V_{1}$ and $V_{2}$, where $V_{1}$ contains speech data and $V_{2}$ contains image data, see section $4 . V_{0}$ characterizes the existence of patterns, see below. Thus, the NMF training problem can be envisaged as:

$$
\left[\begin{array}{l}
V_{0}^{t r n} \\
V_{1}^{t r n} \\
V_{2}^{t r n}
\end{array}\right] \approx\left[\begin{array}{l}
W_{0} \\
W_{1} \\
W_{2}
\end{array}\right] H^{t r n}
$$

We assume $V_{0}$ to be unobservable in the test set. It can then be predicted from the known parts $V_{1}^{t s t}$ and $V_{2}^{t s t}$ for a given $W$, by solving the problem

$$
H^{*, t s t}=\arg \min _{H} D_{K L}\left(\left[\begin{array}{c}
V_{1}^{t s t} \\
V_{2}^{t s t}
\end{array}\right] \|\left[\begin{array}{l}
W_{1} \\
W_{2}
\end{array}\right] H\right)
$$

which is obtained by iterating eq. (3) with both $W_{1}$ and $W_{2}$ kept constant. If we assume the columns in $\left[V_{1}^{t s t} V_{2}^{t s t}\right]^{T}$ are approximately a weighted addition of the same patterns as those that make up the columns of $\left[V_{1}^{t r n} V_{2}^{t r n}\right]^{T}$, every column of $H^{t s t}$ will indicate the extent to which each of those patterns is present in the respective columns of $\left[V_{1}^{t s t} V_{2}^{t s t}\right]^{T}$. Therefore, the data that is missing in the test set can be reconstructed by calculating:

$$
A^{t s t}=W_{0} H^{t s t} \approx V_{0}^{t s t}
$$

In this paper we consider the case where the data in $V_{0}^{t r n}$ and $W_{0}$ are artificially constructed in the following way: suppose there is a set $\mathcal{G}=\left\{p_{1}, p_{2}, \ldots, p_{K}\right\}$, containing $K$ patterns for which the presence or absence in each of the columns of $\left[V_{1}^{t r n} V_{2}^{t r n}\right]^{T}$ is known. $V_{0}^{t r n}$ is then defined as a $K \times N$ matrix for which holds:

$$
V_{0}^{t r n}(i, j)= \begin{cases}1 & \text { if column } \mathrm{j} \text { contains } p_{i} \\ 0 & \text { otherwise }\end{cases}
$$

The inner dimension of the factorization, $R$, is chosen at initialization time to equal the number of detectable patterns $K$. $W_{0}$ is then initialized as the $R \times R$ identity matrix, $I_{R}$. Including $V_{0}$ and $W_{0}$ in the training can be seen as supervision, making solutions where each pattern in $\mathcal{G}$ is assigned to a single column of $W_{1}$ much more likely. This way, it ensures that the presence of any of the $K$ patterns in the columns of $\left[V_{1}^{t s t} V_{2}^{t s t}\right]^{T}$ can be detected by thresholding the columns of A.

This NMF-based framework thus yields base vectors within the columns of the $W$-matrix that consist of a concatenation of a vectorized speech pattern and a vectorized image pattern. Such a column in $W$ can therefore be interpreted as a cross-modal representation of a recognizable object in the data. The semantic link that binds this image pattern and this speech pattern together is established by the fact that both reside in the same column of $W$.

\section{ROW SCALING}

The extended KL-divergence between two sets of values $A$ and $B$ is directly proportional to the mean value of $A$, i.e. for any real value $\alpha$ holds:

$$
D_{K L}(\alpha A \| \alpha B)=\alpha D_{K L}(A \| B)
$$

This means the divergence in equation 1 , which is the sum of divergences of the individual rows in $V$ and $(W H)$, is actually a weighted sum. The weight of each term is proportional to the mean of the elements in the corresponding row of $V$. As a consequence, the mean of a row is proportional to its impact on the factorization.

However, the importance of a row is not necessarily related to its mean. For instance, adding random noise to a row increases its mean, while decreasing its relevance. We therefore surmise that better factorizations, i.e. leading to better predictions, can be achieved by scaling rows appropriately. We introduce the positive diagonal matrix $S$, used for scaling the rows of $V_{1}$. The criterion over which we optimize $S$ is the accuracy of a prediction like the one described in section 2. This prediction cannot be that of $V_{0}^{t r n}$, since the algorithm then settles on the trivial solution where all rows of $V_{1}^{t r n}$ are scaled to zero. Instead we define a set of held out data $V^{x}$ to perform cross-validation. Concretely, we have

$$
\begin{gathered}
\left\{W_{0}^{*}, W_{1}^{*}, H^{* t r n}\right\}= \\
\arg \min _{W_{0}, W_{1}, H^{t r n}} D_{K L}\left(\left[\begin{array}{c}
V_{0} \\
S V_{1}^{t r n}
\end{array}\right] \|\left[\begin{array}{l}
W_{0} \\
W_{1}
\end{array}\right] H^{t r n}\right)
\end{gathered}
$$

which is solved by iterating eq. (2) and (3) for $N^{\text {trn }}$ training iterations. To evaluate on the cross-validation, we first compute $H^{* x}$ :

$$
H^{* x}=\arg \min _{H^{x}} D_{K L}\left(S V_{1}^{x} \| W_{1}^{*} H^{x}\right)
$$


and is solved by iterating eq. (3) for $N^{t s t}$ testing iterations. In all the experiments of this paper, $N^{t r n}$ and $N^{t s t}$ were both set at 100 . Let us denote $\theta=\left\{W_{0}, W_{1}, H^{t r n}\right.$ and $\left.H^{x}\right\}$. If we define $\theta^{\text {init }}$ as its initial value its value after solving (8) and (9), denoted as $\theta^{*}$, then depends on $\theta^{i n i t}, N^{t r n}, N^{t s t}$ and, most importantly, $S$. We can write:

$$
\theta^{*}=f\left(\theta^{i n i t}, N^{t r n}, N^{t s t}, S\right)
$$

After that, the accuracy of the prediction $A^{* x}=W_{0}^{*} H^{* x}$, can be quantified by calculating the Frobeniusnorm

$$
g\left(\theta^{*}\right)=\left\|V_{0}^{x}-\gamma^{*} A^{* x}\right\|_{F}
$$

where

$$
\gamma^{*}=\frac{\sum_{i j} V_{0, i j}^{x} \cdot A_{i j}^{* x}}{\sum_{i j} A_{i j}^{* x} \cdot A_{i j}^{* x}}
$$

We use a Euclidean distance measure here, since the KLdivergence only measures the accuracy with which non-zero values are predicted. The scale factor $\gamma^{*}$ minimizes this distance. Because of this scale factor, the main objective of the rowscaling algorithm, i.e. the equality in equation 6 is relaxed. It now suffices that $A$ is proportional to $V_{0}^{*}$.

We apply $n$-fold cross-validation, defining in each fold $i$ a different training set $V^{(i), t r n}$ and a different cross-validation set $V^{(i), x}$ and using them to calculate the Euclidean distance $g\left(\theta_{i}^{*}\right)$. The optimization of $S$ is then

$$
S^{*}=\arg \min _{S} \sum_{i=1}^{n} g\left(f\left(\theta_{i}^{i n i t}, N^{t r n}, N^{t s t}, S\right)\right)
$$

Eq. 12 naturally leads to an iterative algorithm where for each iteration $S$ is adjusted and the NMF algorithms of eq. (8) and (9) are performed. The optimization of $S$ is a highdimensional box constrained optimization problem. Provided that the gradient $\nabla_{S} g\left(f\left(\theta_{i}^{\text {init }}, N^{t r n}, N^{t s t}, S\right)\right)$ can be calculated, a local optimum can readily be found with dedicated algorithms, such as the L-BFGS-B method [9], which was used in the experiments below. Computationally and algorithmically, the calculation of this gradient is quite involved, but feasible. Due to space constraints, however, we are unable to discuss it in any detail. In all experiments of this paper, the diagonal values of $S$ were initially set to unity.

\section{EXPERIMENTS}

\subsection{Scale-Invariant Feature Transform (SIFT)}

To represent the contents of images in a vectorized way, we make use of the Scale-Invariant Feature Transform [10]. This representation is very well-known and widely used in image processing and recognition. The SIFT-algorithm analyzes a gray-scale image and based on a difference-of-gaussian (DoG) filtering it determines a set of so-called keypoints, i.e. points around which descriptive parts of the image can be found, see figure 1. These parts are converted into "descriptors", 128-dimensional vectors that distinctively describe the

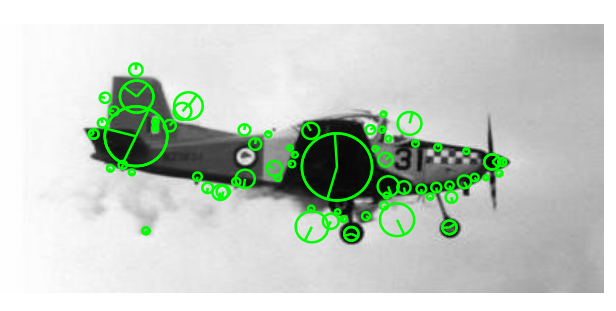

Fig. 1. An example of an image in which the keypoints discovered by the SIFT-algorithm are indicated with circles

area around a keypoint in a scale- and orientation-invariant way. In order to label each of these 128-dimensional vectors, they can be clustered with an algorithm like k-means or KNN, using a codebook of $\mathcal{N}$ prototypes. The descriptors retrieved from a certain image can thus be labelled, counted and placed in a $\mathcal{N}$-dimensional histogram. Histograms of this kind can readily be used as columns in a data-matrix $V$ and decomposed into non-negative base vectors. For the purpose of this paper, we have made use of the open-source library VLFeat [11] to perform these operations.

\subsection{The Histogram of Acoustic Co-occurrences (HAC)}

In order to represent speech utterances in a vectorized way, we make use of HAC [12]. It is similar to SIFT in that it attempts to find discrete labelled acoustic events in the utterance and accumulate them in a histogram. Contrary to the keypoints in images, these acoustic events form a well-defined sequence, such that information about the relations between these events can readily be used. This is done by accumulating co-occurrences of acoustic events in the histogram, instead of just occurrences. An analogy can be drawn with word bigrams used in document clustering and topic detection. A drawback of this approach is that such histograms have a length that equals the square of the number of possible different acoustic events which quickly leads to very high dimensional vectors. The HAC-representation of an utterance is approximately the sum of the HAC-representations of its constituent words, making it useful for analysis of speech with NMF. The HAC-representation has proven to be very suitable for the detection of keywords in spoken utterances and combining different knowledge sources about speech (i.e. different kinds of acoustic events) in an elegant way $[12,13]$.

\subsection{Data}

The visual data in our experimentes consists of images taken from a well-known freely available image database called Caltech 256 [14]. This database consists of a large number of images, each of which belongs to one of 256 different classes. All the images in the same class depict examples of the same object. For the purpose of this paper, we have selected the 10 classes of images from this database that contained the highest number of images. This selection can be seen in table 1 . 


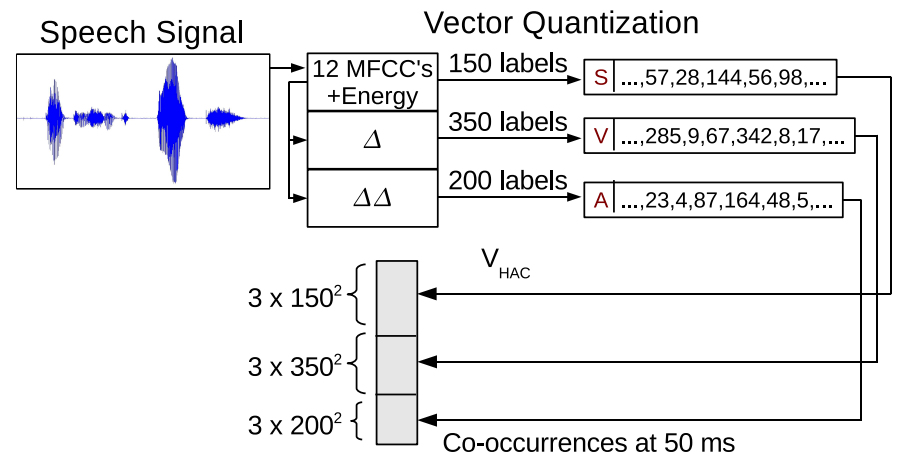

Fig. 2. A schematic overview of the derivation of HACfeatures. The speech signal is converted into three label sequences representing static spectral information $(S)$, velocity information $(V)$ and acceleration information (A). HACvectors are created of each and the three resulting vectors are concatenated.

The SIFT-algorithm was run on these images, yielding a set of 128-dimensional descriptors for every image. These vectors are then divided into 1000 clusters using the k-means algorithm. All interest points in the data are then assigned to one of these 1000 clusters and labelled accordingly. The result for each image is a bag of sift-labels, from which a sparse 1000-category histogram representing the image content can be calculated, that represents the contents of that image.

For the speech data, we make use of a selection from a set of English utterances recorded within the ACORNS project [15], spoken by 10 different speakers. The sentences are syntactically simple, e.g. "Do you see the red ball?". Each utterance contains a single keyword chosen from a set of 10 , corresponding to one of the objects in the images, see table 1 . We analyse each of these selected utterances as follows: we consider $20 \mathrm{~ms}$ signal analysis windows with a frame shift of $10 \mathrm{~ms}$. We calculate 12 MFCC's and the log energy in each of these frames to describe its static spectral content. First and second order differences of these 13-dimensional vectors are used to acquire velocity and acceleration information in each frame. $\mathrm{K}$-means clustering is then performed on these three information streams (statics, velocities and acceleration) to establish a vector codebook for each, that allows us to perform vector quantization of these 13-dimensional "speech descriptors". The codebook sizes for these three streams are 150 for the statics, 350 for the velocities and 200 for the accelerations. The resulting 3 sequences of VQ-labels that thus describe the signal, can be used as discrete acoustic events, the co-occurrences of which can be placed in a histogram. We consider in our experiments only co-occurrences at a time lag of $50 \mathrm{~ms}$ (four intervening labels). This leads to three sparse HAC-vectors of length $150^{2}$ for the statics, $350^{2}$ for the velocities and $200^{2}$ for the accelerations. Concatenated, this yields a vector of length 185000 for every utterance. This process is schematically shown in figure 2 .

The images and speech utterances were chosen in such a way that an artificial semantic link can be established between the 10 recognizable keywords in the speech data and the 10 recognizable objects in the image data. The fact that these semantic links make little sense from a real-world perspective (e.g. airplanes have very little to do with being happy) is not problematic, since the goal of the learning framework is merely to illustrate that the method can learn these semantic connections, whether they make sense or not. Finally, the HAC-vectors and the SIFT-vectors for the corresponding images and utterances are concatenated and placed as the columns in a sparse $V$-matrix with dimension $186000 \times 3914$. From the columns of this matrix 75\% (2936 columns) is randomly selected as the training data $V^{t r n}$, the remaining $25 \%$ (978 columns) is used as test data $V^{t s t}$. Since this data, especially the speech data, is extremely sparse, there are many rows of $V^{t r n}$ that contain only zeros. These rows do not affect the training and can therefore be safely removed. The corresponding rows in $V^{t s t}$ are also removed, even if these do contain non-zero elements. Of the 185000 rows of speech data only 120134 remain after this operation. In the image data all 1000 rows remain. Since the average size of the elements in $V$ heavily influences the result of the factorization, we first normalize this data. If we define the supervision part, the speech part and the image part of $V^{t r n}$ as $V_{0}^{t r n}, V_{1}^{t r n}$ and $V_{2}^{t r n}$ respectively (see section 2), we ensure that in the training set:

$$
\sum_{i j} V_{0, i j}^{t r n}=\sum_{i j} V_{1, i j}^{t r n}=\sum_{i j} V_{2, i j}^{t r n}
$$

and for the test set:

$$
\sum_{i j} V_{1, i j}^{t s t}=\sum_{i j} V_{2, i j}^{t s t}
$$

The reason why $V_{0, i j}^{t s t}$ is left out of this normalization is that the only usable information in this matrix are the positions of the non-zero elements, which are unaffected by such a normalization. This normalization is a preprocessing step performed on all the data and is unrelated to the scaling of the individual input features, which is performed in a later stage (see below).

For the optimization of $S$, we perform five-fold crossvalidation as explained in section 3 on the data in $V^{t r n}$. In each fold $i$ we make a selection of 2349 utterances from $V^{t r n}$ which are used as the set $V^{(i), t r n}$. The cross-validation set $V^{(i), x}$ in that fold then consists of the remaining 587 utterances. These sets are constructed such that the crossvalidation sets in the five folds are disjoint.

\subsection{Experiment}

We performed 6 prediction experiments as described in section 2. The difference between these experiments was the data used and whether the inputs were scaled or not. In short, we did scaled and unscaled recognition using $V_{1}$ (speech), $V_{2}$ (image) and $\left[V_{1} V_{2}\right]^{T}$ (speech and image combined). In each of the experiments, the number of columns in $W$ was set at 10 


\begin{tabular}{|c|c|c|}
\hline number & image classes & keywords \\
\hline 800 & airplane & happy \\
798 & motorcycle & sad \\
435 & faces-easy & dirty \\
358 & t-shirt & clean \\
285 & hammock & round \\
278 & billiards & square \\
270 & horse & yellow \\
242 & ladder & blue \\
232 & bathtub & red \\
216 & binoculars & small \\
\hline 3914 & \multicolumn{2}{|c|}{ Total } \\
\hline
\end{tabular}

Table 1. The selection of image categories from Caltech 256 and keywords from the ACORNS database and the way they are semantically mapped onto each other.

to accommodate representations for the 10 objects/keywords. Because NMF is prone to converging towards local optima, and $W_{1}$ and $H^{t r n}$ are always randomly initialized, the prediction $A$ is not deterministic. Therefore, in each experiment the algorithm was run 5 times, each time with a different random initialization and yielding a slightly different activation matrix $A$.

For the optimization of $S$ in each experiment, an initial value for $W, H^{t r n}$ and $H^{x}$ was made in every one of the five folds. The inner dimension of the factorization $R$ was here also set to 10. In each fold $i$, unscaled NMF training was performed for 100 training iterations and 100 testing iterations to determine the starting point $\theta_{i}^{\text {init }}$. $S$ was then once more optimized as explained in section 3. $N^{t r n}$ and $N^{t s t}$ were both set at 100. The optimization of $S$ was run for 30 iterations. At this point, convergence was not reached yet, but closely approximated. To demonstrate the effect of the algorithm on the data we have analyzed the scaling of the HAC-features in the speech experiment, see figure 3 . The top figure shows the 100 features with the largest mean values in $V_{1}^{t r n}$, i.e. the ones having the highest impact on the unscaled result. Both the original mean values and the values after scaling are shown. The bottom figure shows the reverse, i.e. the 100 features that have the highest value after scaling and their corresponding value before scaling. These plots show that the main benefit of the algorithm is that it scales down "bad" features that affect the result but does not scale up "good" features with little influence. Finally, when $S^{*}$ is known, it is applied on the rows of the complete data set, giving $S^{*} V_{1}^{t r n}$ as train set and $S^{*} V_{1}^{t s t}$ as test set. The training and testing procedure on this scaled data is then repeated five times, using different random initializations.

\subsection{Results}

If we make use of the knowledge that there is only a single keyword/object to be recognized in each utterance/image of the test set, recognition can be achieved by determining the highest value in each column of the prediction matrix $A$. We
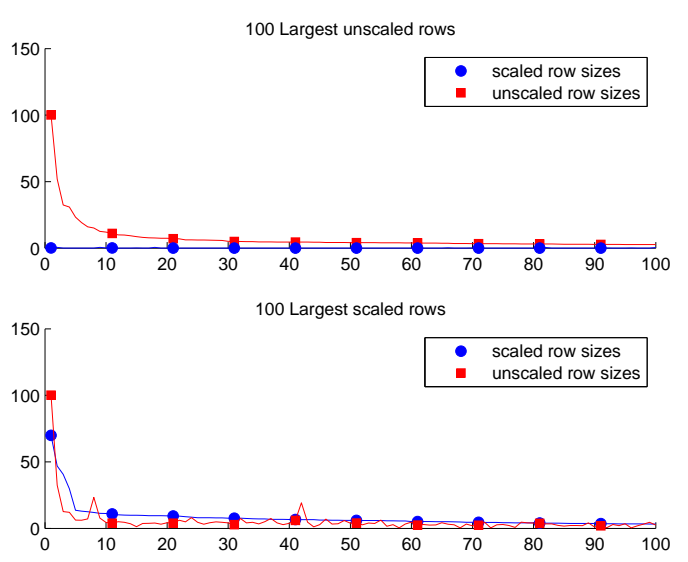

Fig. 3. The top figure shows the mean values of the largest rows in $V_{1}^{\text {trn }}$ of the speech experiment before scaling along with their mean after scaling. The bottom figure shows the same but now for the largest rows after scaling

\begin{tabular}{|c|c|c|}
\hline \multirow{2}{*}{} & \multicolumn{2}{|c|}{ OKER(\%) } \\
\cline { 2 - 3 } & No Scaling & Scaling \\
\hline \hline speech & 8.58 & $\mathbf{1 . 3 3}$ \\
image & 41.52 & $\mathbf{3 6 . 1 6}$ \\
speech + image & 20.53 & $\mathbf{6 . 8 4}$ \\
\hline
\end{tabular}

Table 2. The object/keyword error rates in all six recognition experiments.

can then count the percentage of utterances/images in the test set for which this prediction is wrong. Obviously, the lower this object/keyword error rate (OKER), the more accurate the acquired models are. This way of evaluating the learning framework is very insensitive to the randomness of the initialization of NMF. In none of our experiments, the initialization in the five runs made a difference for the eventual result. The results of all experiments are shown in table 2.

Note that for the experiment with speech and image data combined, a superior result would be achieved if the scale factors of $V_{2}$ were all set to zero and those of $V_{1}$ were set equal to those found in the experiment with only speech. The reason why this solution is not found is probably because the row scaling algorithm only guarantees a local optimum, not a global one, since the objective function 11 is not convex over $S$.

\section{DISCUSSION}

From the results in table 2 it is clear that recognizing the 10 objects from the image data is a much harder problem than recognizing the 10 keywords from the speech data. Although one might expect that adding speech and image data together would yield better results than either of them separately, these experiments show that this is not the case. At least for the rather naive way these two modalities are combined here. 
More advanced means of combining information from different modalities are described in literature $[3,1]$.

The novelty of the work presented here, however, is the enhancement of the NMF-based learning framework brought about by the input scaling algorithm, which is demonstrated by a reduction of the error rate in every experiment performed. These improvements are probably due to the nature of both SIFT- and HAC features. First, these features carry information about everything in the utterance/image, whether it relates to the keyword/object to be recognized or not. A large number of features is therefore useless. A second reason is that some features may be relevant but not distinctive for one particular keyword/object, in which case they do not aid recognition. Aside from enhancing recognition results, the optimal weights $S^{*}$ are an important tool if one wants to reduce the number of input features by performing feature selection. This is important, especially for the HAC features, since they are so high-dimensional. Instead of removing feature $i$ if $\sum_{j} V_{i j}$ is too small, a better criterion for removing feature $i$ is to check whether $\sum_{j} s_{i}^{*} V_{i j}$ is small.

Note that this scaling algorithm is not exclusively applicable to keyword or image recognition. It can be applied on any supervised learning problem that is solved with NMF, and by extension pLSA, since it is virtually the same algorithm [16].

\section{CONCLUSION}

In conclusion, we have shown that the NMF-paradigm can be improved. Each of its individual input features exercises an amount of influence over the result that is not necessarily proportional to its merit. We have therefore introduced an algorithm that attempts to scale the input features of NMF appropriately, in order to alleviate this shortcoming. We have shown that this algoritm consistently improves the recognition results in problems where one is forced to resort to learning with NMF, such as object recognition in images and multimodal learning from speech and images.

\section{ACKNOWLEDGMENTS}

This research was part of the VASI project (Research fund K.U.Leuven OT/09/028).

\section{REFERENCES}

[1] K. Saenko and T. Darrell, "Object category recognition using probabilistic fusion of speech and image classifiers," in proc. MLMI, Brno, Czech Republic 2007.

[2] Nakamura T., Nagai T., and Iwahashi N., "Grounding of word meanings in multimodal concepts using lda," in proc. International Conference on Intelligent Robots and Systems, October 2009.
[3] R. Lienhart, S. Romberg, and E. Hörster, "Multilayer plsa for multimodal image retrieval,' in proc. CIVR '09, July 2009.

[4] D.D. Lee and H.S. Seung, "Learning the parts of objects by nonnegative matrix factorization," Nature, , no. 401, pp. 788-791, 1999.

[5] Thomas Hoffman, "Probabilistic latent semantic analysis," in proc. UAI, Stockholm, 1999.

[6] David M. Blei, Andrew Y. Ng, and Michael I. Jordan, "Latent dirichlet allocation," Journal of Machine Learning Research, , no. 3, pp. 993-1022, 2003.

[7] V. Y. F. Tan and C. Févotte, "Automatic relevance determination in nonnegative matrix factorization," in proc. Workshop Signal Process. Adaptative Sparse Structured Representations (SPARS'05), Saint-Malo, France, april 2009.

[8] M. Mørup and L. K. Hansen, "Tuning pruning in sparse non-negative matrix factorization," in European Signal Processing Conference 2009 (EUSIPCO'09), 2009.

[9] R. H. Byrd, P. Lu, and J. Nocedal, "A limited memory algorithm for bound constrained optimization," SIAM Journal on Scientific and Statistical Computing, vol. 16, no. 5, pp. 1190-1208, 1995.

[10] David G. Lowe, "Object recognition from local scaleinvariant features," in proc. International Conference on Computer Vision, 1999, pp. 1150-1157.

[11] A. Vedaldi and B. Fulkerson, "vlfeat: An open and portable library of computer vision algorithms," http://www.vlfeat.org/, 2008.

[12] Hugo Van hamme, "HAC-models: a novel approach to continuous speech recognition," in proc. Interspeech, Brisbane, Australia, september 2008, pp. 2554-2557.

[13] H. Van hamme, "Integration of asynchronous knowledge sources in a novel speech recognition framework," in proc. ITRW on Speech Analysis and Processing for Knowledge Discovery, Aalborg, Denmark, 2008.

[14] G. Griffin, A. Holub, and P. Perona, "Caltech-256 object category dataset," Tech. Rep. 7694, California Institute of Technology, 2007.

[15] "Acorns: Acquisition of communication and recognition skills," http://lands.let.ru.nl/acorns/about.html, 2006-2009.

[16] E. Gaussier and C. Goutte, "Relation between plsa and nmf and implications," in proc. SIGIR, Salvador, Brazil, 2005. 